\title{
Unexpected anatomical situs after surgery for vesicoureteral reflux
}

Haemmerle Beatrix, Schmid Seraina, Aurbach Kathrin

Gynecology and Obstetrics, Hospital Grabs, Grabs, Switzerland

\section{Introduction:}

We present the case of a 52 year old woman with a history of repeat surgery for vesicorenal reflux (VUR) undergoing elective hysterectomy.

\section{Case report:}

After repeat urinary tract infection (UTI), VUR was diagnosed. At 8 years old, a Lich-Gregoir

ureteroneocystostomy was performed on the left side, creating an extravesical tunnel for the ureter.

Two years later, having been diagnosed with bilateral VUR the patient underwent a double-sided PolitanoLeadbetter ureteroneocystostomy. This technique aims for an anatomically correct reinsertion of the ureter. A subsequently performed intravenous pyelogram revealed hydronephrosis and hydroureter on the right side. In the following years, no further therapy was needed. The patient had five spontaneous deliveries after uncomplicated pregnancies. At the age of 37 , an isotope nephrography performed for flank pain showed a limited function of the right kidney of $14 \%$.

Nephrectomy was evaluated but finally rejected since patient's symptoms improved after treatment of UTI. At the age of 52 , suffering from menometrorrhagia following normal findings in dilatation and curettage the patient was counselled for laparoscopic hysterectomy and salpingectomy. During this procedure, a doubling of the broad ligament adjacent to the uterus on the right side was severed whereas the rest of the operation went without abnormalities.

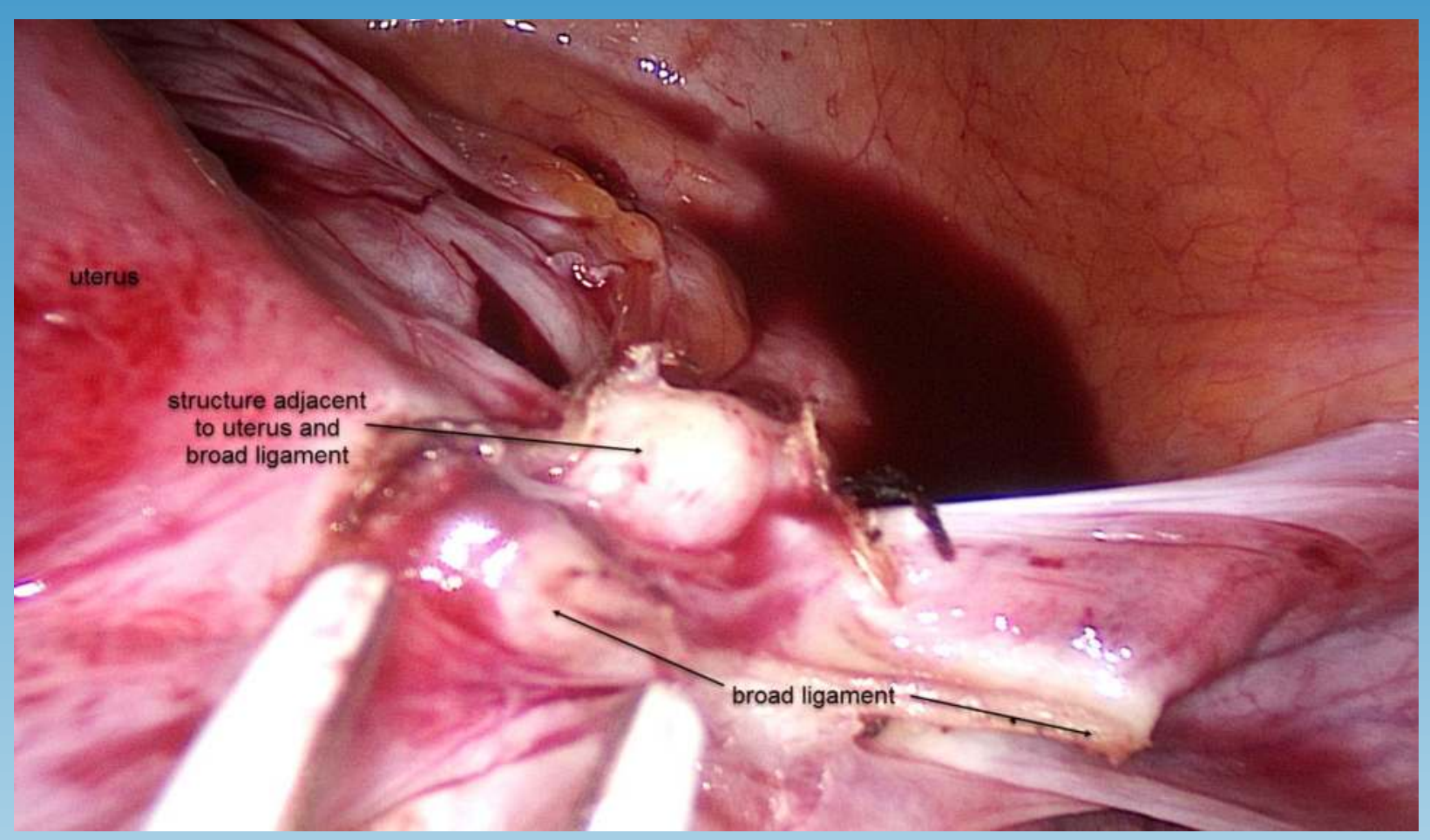

At the end of the operation it was noted that the severed structure had held the kinked right ureter.

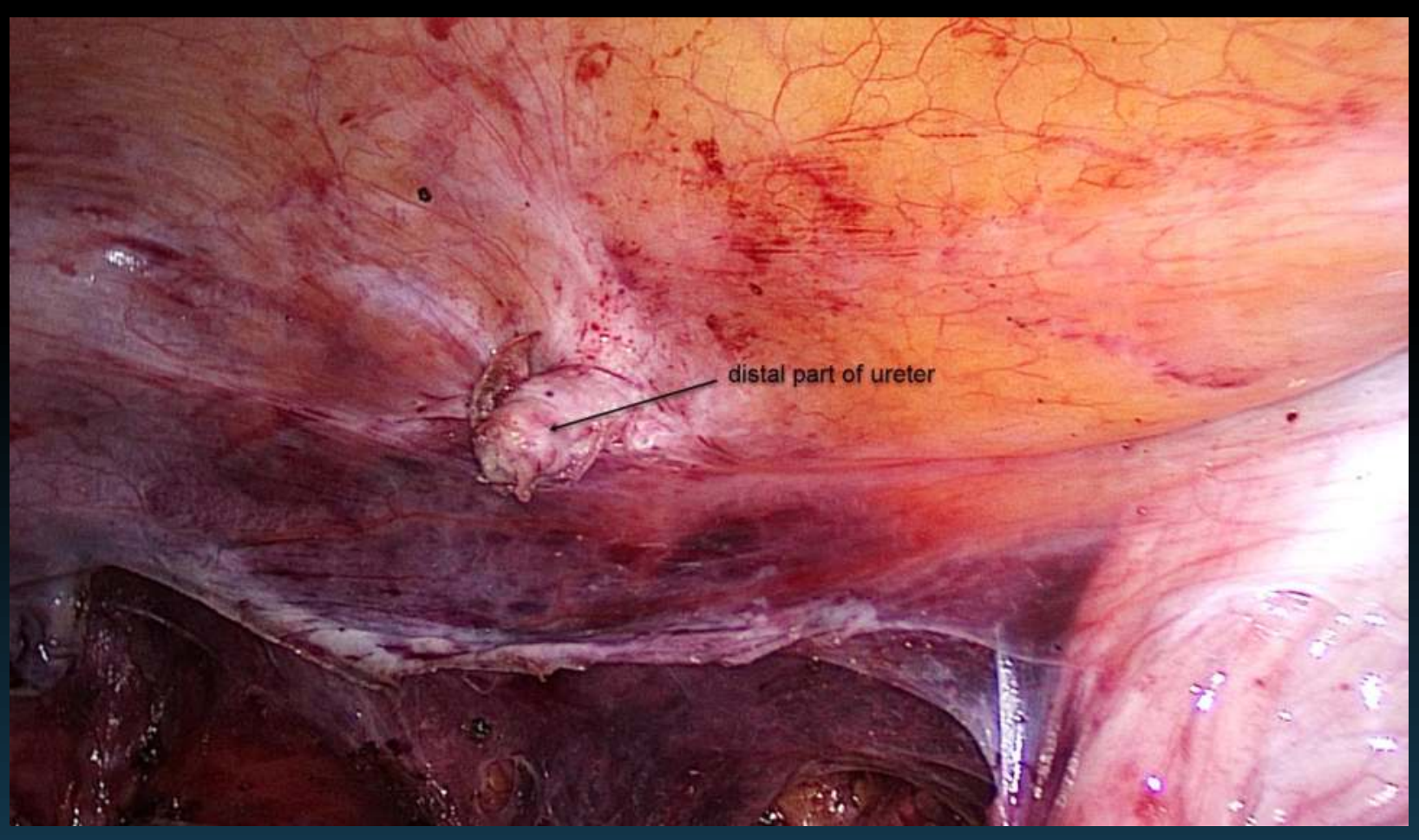

Direct reanastomosis couldn't be performed due to distance between the two parts. Therefore and in the knowledge of limited kidney function on the right side the team decided to clip both proximal and distal ureter parts.

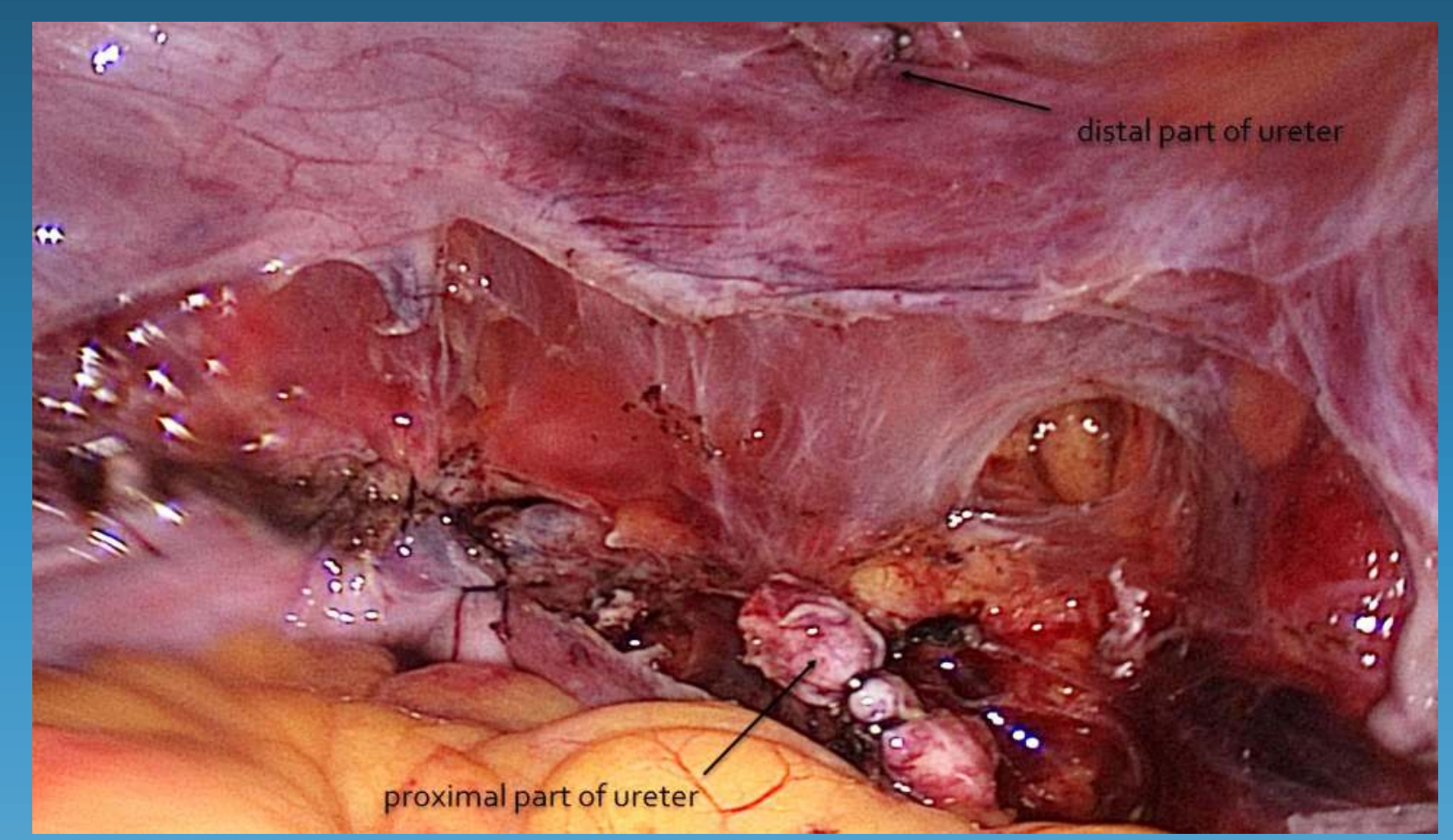

After developing hydronephrosis, the patient received a nephrostomy on the right side.

Since an intravenous pyelogram confirmed the limited function of the right kidney, laparoscopic nephrectomy was performed.

\section{Conclusion:}

Although confined to the intramural part, surgery for VUR can also affect the more proximal part of the ureter. In order not to complicate routine surgery, changes in 